\title{
The Relationship Between Human Papillomavirus, OFDI and Primary Ciliogenesis in the Progression of Oropharyngeal Cancer: A Retrospective Cohort Study
}

This article was published in the following Dove Press journal:

Pharmacogenomics and Personalized Medicine

\author{
Hong-xue Meng (iD) ${ }^{1,2 *}$ \\ Xin-xin Yang ${ }^{1}{ }^{1, *}$ \\ Rui-qi Liu ${ }^{3, *}$ \\ Jun-jie Bao ${ }^{4}$ \\ Yun-jing Hou iD ' \\ Ji Sun ${ }^{5}$ \\ Su-sheng Miao ${ }^{5}$ \\ Guo-fan $\mathrm{Qu}^{4}$ \\ 'Department of Pathology, Harbin \\ Medical University Cancer Hospital, \\ Harbin, People's Republic of China; \\ ${ }^{2}$ Department of Pathology, Harbin \\ Medical University, Harbin, People's \\ Republic of China; ${ }^{3}$ Department of \\ Radiation Oncology, Sun Yat-Sen \\ University Cancer Hospital, Guangzhou, \\ People's Republic of China; ${ }^{4}$ Department \\ of Orthopedics, Harbin Medical \\ University Cancer Hospital, Harbin, \\ People's Republic of China; ${ }^{5}$ Department \\ of Otolaryngology, Head and Neck \\ Surgery, Harbin Medical University \\ Cancer Hospital, Harbin, People's \\ Republic of China
}

*These authors contributed equally to this work

Correspondence: Hong-xue Meng Department of Pathology, Harbin Medical University Cancer Hospital, 150 Haping

Road, Harbin, People's Republic of China Tel +86-45I-857|826I

Email menghongxuel5@163.com

Guo-fan Qu

Department of Orthopedics, Harbin

Medical University Cancer Hospital, I50

Haping Road, Harbin, People's Republic of

China

Tel +86-45I-857I8262

Email188467I9333@I63.com
Purpose: Infection with human papillomavirus (HPV) has been indicated to be a important risk factor for oropharyngeal squamous cell carcinoma (OPSCC). Primary ciliogenesis defects contribute to tumorigenesis, and OFD1 at centriolar satellites is a crucial suppressor of primary ciliogenesis. To identify novel markers associated with HPV-induced carcinogenesis, the interactions between HPV infection and primary ciliogenesis in the tumorigenesis and progression of OPSCC were investigated in this study.

Patients and Methods: The 1530 OPSCC patients recruited in this research were treated from 2000 to 2017. Immunohistochemistry and RT-PCR were performed on tissue samples to compare the expression of p16, TSLP, TGF $\beta 1$, IFN $\gamma$, OFD1, and their relationship with clinical characteristics of patients.

Results: We speculate that the positive expression of p16 is related to early primary OPSCC, and the survival rate of $p 16$ positive patients after radiotherapy and surgery is higher. Expression of TSLP on dendritic cells in HPV-positive OPSCC correlated with the expression of OFD1. HPV-positive OPSCC showed increased expression of OFD1 combined with reduced ciliogenesis. Hence, TSLP induced by HPV infection may reduce the invasive potential of OPSCC cells by promoting OFD1 expression, thereby inhibiting primary ciliogenesis.

Conclusion: Our study demonstrated that HPV may be related to the progression of OPSCC by regulating OFD1 expression and primary ciliogenesis, making this protein a potential therapeutic target.

Keywords: HPV, p16, oropharyngeal cancer, pathogenesis, prognosis

\section{Introduction}

Head and neck cancer was the seventh most common cancer worldwide, and the proportion of Oropharyngeal squamous cell carcinoma (OPSCC) in head and neck cancer is increasing year by year., which is characterized by nodal metastases and high recurrence rates. ${ }^{1}$ OPSCC can usually be suppressed by surgery and adjuvant radiotherapy in the early stages of development, but the 5-year survival rate in locally advanced patients is only $48 \%$, and only $26 \%$ in patients with metastatic disease. . $^{2,3}$

Studies have confirmed that high-risk factors for head and neck squamous cell carcinoma (HNSCC) include tobacco, alcohol, and poor oral hygiene. However, the 
incidence of these risk factors is declining in recent years. ${ }^{3}$ Human papillomavirus (HPV) infection has become a important high-risk factor for HNSCC. Including the United States, Australia and some European countries, the prevalence of HPV-related HNSCC, especially OPSCC has been rising. ${ }^{4,5}$ Nonetheless, in the Chinese patient cohort, the prevalence, prognosis and significance of OPSCC caused by high-risk HPV infection still remain underexplored.

The current rising in OPSCC is mainly related to highrisk HPV infection. ${ }^{2} 90 \%$ of HPV-positive OP cancers are infected with high-risk HPV16 (the dominant subtype). ${ }^{6}$ In cancers associated with HPV infection, the viral oncoproteins E6 and E7 are always overexpressed. E7 can bind to the retinoblastoma $(\mathrm{Rb})$ protein and destroy the $\mathrm{E} 2 \mathrm{~F} / \mathrm{Rb}$ complex. After Rb protein is degraded, cell cycle limitation is eliminated and transcription factor E2F is associated with increased p16 (INK4) protein expression. ${ }^{7}$

HPV infection is closely related to $\mathrm{p} 16$ protein expression in OPSCC. Therefore, in these high-risk tumors, p16 can be used as a surrogate biomarker to detect the presence of HPV infection. ${ }^{9,10} \mathrm{HPV}$ is transcribed before p16 expression. Therefore, p16 expression can be used as an indicator to identify tumors with HPV transcription activity. ${ }^{11}$ Moreover, compared with the use of histochemical methods to detect HPV DNA, p16 expression can be used as an independent prognostic factor for overall survival and progression-free survival. ${ }^{12}$ Then, p16 immunohistochemistry (IHC) in combination with HPV-DNA detection by PCR has been found to be a powerful indicator of clinical outcome in patients with OPSCC using univariate analysis. ${ }^{7,13}$ And previous studies have indicated that p16 may enhance the immunogenicity of dendritic cells (DC) by means of Th1 cytokine secretion and cyclin-dependent pathways. $^{8}$

HPV infection can give rise to a very extensive of epithelial lesions. However, only patients with high-risk types of HPV is possible to transform cancer. ${ }^{14,15}$ The activated thymic stromal lymphopoietin (TSLP) secreted by the head and neck epithelium can bind to the heterodimeric receptor composed of IL-7 receptor alpha chain (IL-7R $\alpha$ ) and TSLP receptor (TSLPR) chain. ${ }^{16,17}$ Exogenous stimulation (trauma, infection, etc.), Toll-like receptor signal transduction, and host-derived pro-inflammatory and Th2 cytokines can all induce TSLP activation. Because TSLP, IFN $\gamma$ and TGF $\beta 1$ can represent the levels of the various components of the immune response, so in our research we tested their content to understand how they are related in tumor immune response.

The proportion of HPV DNA-positive head and neck cancer (HNC) caused by HPV infection in the overall HNC is indetermination, and its estimation remains a formidable challenge. Therefore, it is very significance to explore the carcinogenesis induced by HPV infection and its related expression patterns of individual and combined markers, which can be used to evaluate the biological and carcinogenic activity of HPV in OPSCC. For example, the involvement of TSLP in OPSCC with HPV infection is unknown.

The primary cilium is a organelle based on microtubule structures and play an important role in sensory and signaling pathways. The maintenance of the normal function of many signaling pathways is inseparable from the cilium structure. These signaling pathways play a vital role in the development of many types of cancer.There are abnormalities and defects in the function of primary cilia in human cancer cells, which promotes the occurrence and development of tumors. ${ }^{18,19}$ OFD1 on the centromeric satellite is a key factor used to inhibit the growth of primary cilia in human cancer cells. The growth of mammalian primary cilia is generally performed by removing OFD1 by autophagy. ${ }^{20}$ OFD1 expression could be regulated by DCs. However, it is unknown whether TSLP regulates OFD1 expression and primary ciliogenesis.

This study aims to evaluate the prevalence, prognosis, and clinicopathologic characteristics of HPV-positive oropharyngeal cancer in Northeast China, and to clarify the role of TSLP, p16 and OFD1 in the occurrence and development of OPSCC.

\section{Patients and Methods}

\section{Patients}

One thousand five hundred and thirty patients with pathology-proven oropharyngeal cancer (January 2000 to January 2017) were enrolled from Harbin Medical University Cancer Hospital, which was the cancer center for northeast China. The acquired tissue was taken during the operation of the patient.

\section{Ethics Statement}

This research strictly follows the Helsinki Declaration. This research was approved by the institutional ethics committee of the Cancer Hospital Affiliated to Harbin Medical University. 


\section{Clinical Parameters}

The clinical data of patients include data on gender, age, history of smoking, history of alcohol, treatment.

\section{Histopathological Diagnosis}

The diagnosis and categorize of all cases strictly follow the relevant WHO principles. Two pathologists reviewed all slides and scored the pathological variables. The TNM classification of HPV-positive oropharyngeal cancer was developed by the International Oropharyngeal Cancer Staging Network Cooperation Organization (ICON-S). ${ }^{21,22}$

\section{Antibodies and Immunohistochemistry $(\mathrm{IHC})$}

For IHC, formalin-fixed, the $4 \mu \mathrm{m}$ thick FFPE was blocked with $1 \% \mathrm{H}_{2} \mathrm{O}_{2}$, and then incubated with trypsin at $37{ }^{\circ} \mathrm{C}$ for 30 minutes for antigen retrieval. IHC was performed according to the manufacturer's instructions. Sections were incubated with the following primary antibodies overnight at $4{ }^{\circ} \mathrm{C}$ : rabbit anti-human P16 (1:100, INK4a, IgG, Zhongshan Tech, China), rabbit anti-human TSLP (10 $\mu \mathrm{g} / \mathrm{mL}$, ab47943, IgG, Abcam, USA), goat antihuman TSLPR (10 $\mu \mathrm{g} / \mathrm{mL}, \mathrm{IgG}, \mathrm{R} \& \mathrm{D}$, USA), rabbit antihuman OFD1 (1:100, IgG, Abcam, USA), goat anti-human TGF- $\beta 1(10 \mu \mathrm{g} / \mathrm{mL}, \mathrm{IgG}, \mathrm{R} \& \mathrm{D}$, USA), rabbit anti-human IFN $\gamma$ (1:100, IgG, Abcam, USA), mouse anti-human DCSIGN (1:100, IgG, Abcam, USA). To demonstrate the structures of cilia, we stained them with ARL13B (1:100, IgG, Abcam, USA), a ciliary membrane marker. Sections were then incubated with Streptavidin-Biotin Universal Detection System (Beckman Coulter, USA), and visualized using DAB.

For IHC analysis of p16 expression, cells presenting nuclear and cytoplasmic staining were classified as positive, and were scored semi-quantitatively according to a previous study, ${ }^{23}$ as follows: negative (no cells were positive); sporadic (isolated cells were positive, but $<5 \%$ ); focal (small cell clusters, but $<80 \%$ of the cells were positive); and diffuse $(>80 \%$ of the cells were stained). Strong and diffuse nuclear and cytoplasmic staining in $\geq 80 \%$ of tumor cells was defined as p 16 positive.

\section{In situ Hybridization (ISH)}

For IHC, fix it with formalin, FFPE $4 \mu \mathrm{m}$ thick and seal it, then incubate with HPV probe (1: 100, Abcam).

\section{DNA Extraction and PCR Analysis}

We used the DNeasy Micro kit (Qiagen, Hilden, Germany) to extract and purify total DNA from formalin-fixed paraffin-embedded tissue according to the manufacturer's instructions. The DNA was amplified after 35 cycles for PCR analysis. The forward and reverse primers are: $\beta$ globin 5'- GAA GAG CCA AGG ACA GGT AC -3' (forward) and 5'- CAA CTT CAT CCA CGT TCA CC -3' (reverse); HPV 5'- CGT CCM ARR GGA WAC TGA TC 3' (forward) and 5'- GCM CAG GGW CAT AAY AAT GG $-3^{\prime}$ (reverse). The cycling conditions of HPV and $\beta-$ globin are: the denaturation temperature is at $94{ }^{\circ} \mathrm{C}$ for 1 minute, the annealing temperature is $56{ }^{\circ} \mathrm{C}$ for 1 minute, the extension temperature is $72{ }^{\circ} \mathrm{C}$ for 1 minute, and finally Extend for 10 minutes at $72{ }^{\circ} \mathrm{C}$. The PCR products were analyzed by $4 \%$ agarose gel electrophoresis, and observed on the imager using ethidium bromide.

\section{RNA Extraction and RT-PCR Analysis}

Total RNA was extracted and purified from LCM-captured cells from formalin-fixed, paraffin-embedded tissues using the RNeasy Micro kit (Qiagen, Hilden, Germany) according to the manufacturer's instructions. We used the QuantiTect reverse transcription kit (Qiagen, Hilden, Germany) for the synthesis of complementary DNA (cDNA). The resulting cDNA was used as a template for PCR analysis. Forward and reverse specific primers, amplicon size and annealing temperature are: $\beta$-actin 5'-CAGAGCAAGAGAGGCAT CCT-3' (forward) and 5'-ACGTACATGGCTGGGGTG-3' (reverse), $227 \mathrm{bp}, 55{ }^{\circ} \mathrm{C}$; TSLP $5^{\prime}$-TATGAGTGGGACC AAAAGTACCG-3' (forward) and 5'-GGGATTGAAGGTT AGGCTCTGG-3' (reverse), $97 \mathrm{bp}, 55^{\circ} \mathrm{C}$; TSLPR 5'-GA GTGGCAGTCCAAACAGGAA-3' (forward) and 5'-ACAT CCTCCATAGCCTTCACC-3' (reverse), $103 \mathrm{bp}, 62{ }^{\circ} \mathrm{C}$; TGF- $\beta 1 \quad 5^{\prime}$-ACCAACTATTGCTTCAGCTC-3' (forward) and 5'-TTATGCTGGTTGTACAGGG-3' (reverse), $197 \mathrm{bp}$, $50{ }^{\circ} \mathrm{C}$. The PCR products were analyzed by $4 \%$ agarose gel electrophoresis, and observed on the imager using ethidium bromide.

\section{Quantitative Real-Time PCR Analysis}

We used QuantiTect RT kit (Qiagen, Hilden, Germany) for reverse transcription of the same amount of RNA (50 ng) from the sample. In addition, we use the Fast SYBR Green Master Mix (Applied Biosystems, Germany) for the amplified cDNA, and the 7500 Fast Real-Time PCR System (Applied Biosystems) for PCR analysis samples. Primers 
for $\beta$-actin, TSLP, TSLPR, and TGF- $\beta 1$ were described in the RT-PCR section. We applied the relative standard curve method to clarify its relative expression. And the data was normalized to $\beta$-actin expression.

\section{Statistical Analysis}

In our research, Mann-Whitney $U$-test was performed to compare data between P16 positive and negative patients. We speculated the relationships among clinical characteristics, HPV status, and expression of p16, TSLP, TGF $\beta 1$, IFN $\gamma$, and OFD1 using Spearman correlation analysis and Pearson's correlation analysis (SAS Institute Inc., Cary, NC, USA). Differences with $p$-values of less than 0.05 were considered significant.

\section{Results}

\section{Clinical and Pathological Parameters}

Table 1 shows the clinicopathological characteristics of OPSCC in our cohort (1530 cases). The patients were predominantly male $(\mathrm{n}=1197 ; 78.23 \%)$ and smokers $(\mathrm{n}=1326 ; 86.67 \%) .920$ had a history of drinking $(60.13 \%)$. Treatment consisted in surgery only $(\mathrm{n}=1287)$, postoperative radiotherapy $(\mathrm{n}=235)$, postoperative chemotherapy $(n=5)$, or surgery followed by radiotherapy and chemotherapy $(\mathrm{n}=3)$. We have followup information for all 1530 patients. After completing initial treatment, there are residual diseases in 286 persons (18.69\%). A complete response (CR) was achieved in 1244 patients $(81.31 \%)$, of which $773(50.52 \%)$ maintained CR during the follow-up and the other 471 (30.78\%) subsequently showed recurrence or metastasis.

According to the histological type, there were 204 cases $(13.33 \%)$ in 1530 cases of SCC NOS/conventional non-keratinization treatment, and 1301 cases $(85.03 \%)$ in conventional keratinization (Table 2). Regarding the differentiation, 497 (32.48\%), 848 (55.42\%), and 185 $(12.09 \%)$ cases showed good, moderate, and poor differentiation, respectively. Most patients have lymphatic infiltration $(645,42.16 \%)$.

In the TNM staging, 1455 patients $(95.1 \%)$ had low $\mathrm{T}$ stage OPSCC tumors (T1/T2), and other 75 patients (4.9\%) had high T stage OPSCC tumors (T3/T4); In addition, there were 645 patients $(42.16 \%)$ emerged clinically positive lymph node metastasis $(\mathrm{N}+)$. Finally, 1368 patients $(89.41 \%)$ had a high clinical stage (III/IV), and the remaining 162 patients $(10.59 \%)$ had a low clinical stage (I/II).

\section{PI6 Protein Overexpression, HPV DNA-PCR, and HPV-ISH}

IHC showed that p16 was overexpressed in $81(5.29 \%)$ of these 1530 cases. In addition over $80 \%$ of carcinoma cells and in situ carcinoma cells have strong and diffuse staining in the nucleus and cytoplasm in these p16 positive cases (Figure 1). Among all cases, 78 (5.10\%) were shown to be HPV-positive by PCR (Figure 2). ISH results showed that 38 patients were positive for HPV. Of these, 5 cases were positive for HPV6, 3 positive for HPV11, 2 for HPV13, 25 for HPV16, and 3 for HPV18.

Consistently, there is a good correlation between HPV positive and $\mathrm{p} 16$ overexpression. Overexpression of $\mathrm{p} 16$ as a predictor of HPV infection showed high sensitivity $(100 \%)$ and high specificity (96\%). Table 1 provides a detailed description of the relationship between HPV infection and clinicopathological variables. HPV infection was significantly more frequent in patients who were older $(\mathrm{P}<0.01)$ or male $(\mathrm{P}<0.05)$, had moderate and poor differentiation $(\mathrm{P}<0.05)$, conventional keratinizing type ( $\mathrm{P}<0.01)$, lymph node metastasis $(\mathrm{P}<0.05)$, low $\mathrm{T}$ stage $(\mathrm{P}<0.05)$, high clinical stage $(\mathrm{P}<0.05)$, and $\mathrm{p} 16$ overexpression $(\mathrm{P}<0.01)$. Specifically, HPV and p16-positive patients were more likely to maintain the $\mathrm{CR}$ during the follow-up period.

\section{Relationship Between HPV and Expression of TSLP, TGF $\beta$ I, IFN $\gamma$, and OFDI}

Expression of TSLP, TGF $\beta 1$, and IFN $\gamma$ was higher in OPSCC tissues from HPV-positive than from HPVnegative patients (Figure 3). Among them, their statistical values are as follows: TSLP $(\mathrm{p}<0.05, \mathrm{r}=0.62)$, TGF $\beta 1$ $(\mathrm{p}<0.05, \mathrm{r}=0.65), \quad$ IFN $\gamma \quad(\mathrm{p}<0.05, \mathrm{r}=0.73)$. Furthermore, higher expression of OFD1 combined with less ciliogenesis was observed in HPV-positive OPSCC tissues (Figure 4), which may explain why HPV-positive OPSCC cases showed more frequently moderate and poor differentiation and lymph node metastasis. Correlation analysis by Spearman showed that the expression of TSLP was related to the expression of TGF $\beta 1$, IFN $\gamma$ and OFD1. Using double immunofluorescence, we found that TSLP was overexpressed on DCs in HPV-positive OPSCC, and that TSLP expression on DCs correlated with OFD1 expression (Figure 5). Moreover, the mRNA levels of TSLP, TGF $\beta 1$, IFN $\gamma$, and OFD1 were higher in the HPV-positive than in the HPV-negative group $(\mathrm{P}<0.05$, Figure 6). 


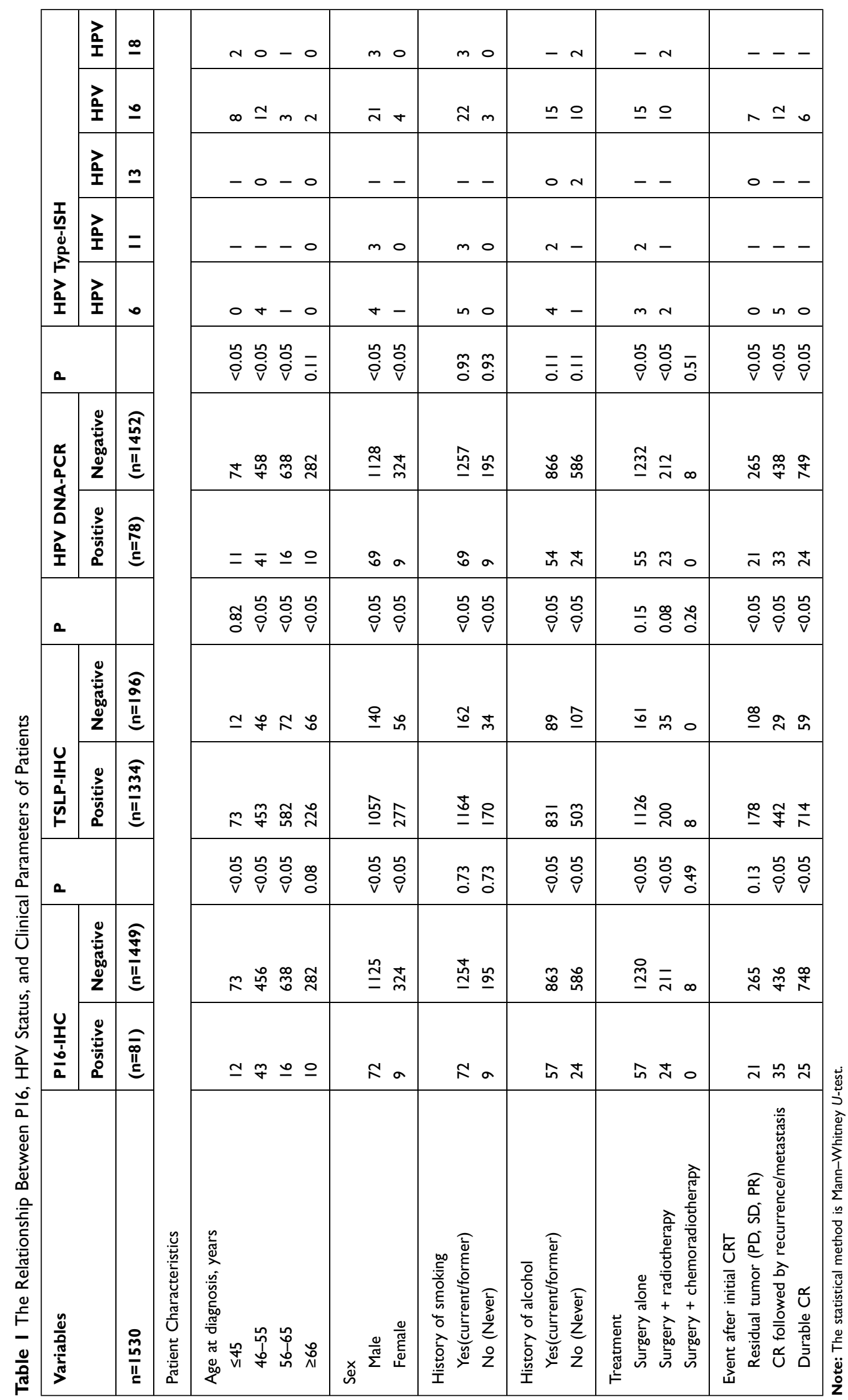




\begin{tabular}{|c|c|c|c|c|c|c|c|}
\hline \multirow{5}{*}{ 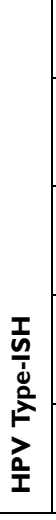 } & $\frac{2}{2}$ & $\underline{\infty}$ & $0 m 000000$ & $n-0 N m-0$ & $0 m 00$ & --1000 & $\circ \mathrm{m}$ \\
\hline & 를 & $\stackrel{\circ}{-}$ & $0 \underline{n}+00000$ & $\sigma \stackrel{m}{m} \underline{a} \stackrel{\sim}{n} 0$ & 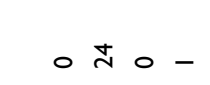 & $0 \infty \infty N-0$ & $0 \stackrel{2}{N}$ \\
\hline & 主 & $\underline{m}$ & -1000000 & $-0-0-00$ & --00 & $\begin{array}{lllllllllllll}n & 0 & 0 & 0 & 0 & 0\end{array}$ & -- \\
\hline & $\frac{2}{1}$ & $=$ & $-n O C O 00000$ & $---N m 00$ & $0 m 00$ & $-0 N 000$ & $\circ \mathrm{m}$ \\
\hline & 亭 & ○ & n 00000000 & 0 in 0 in t 00 & -+00 & $0 m \sim 000$ & -+ \\
\hline \multicolumn{2}{|l|}{ a } & & 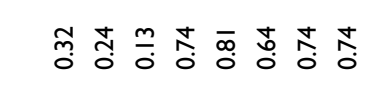 & 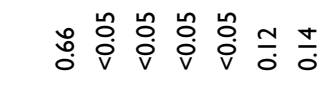 & 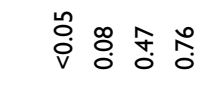 & 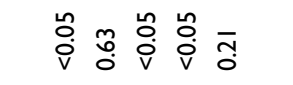 & 苛 \\
\hline \multirow{2}{*}{$\begin{array}{l}\text { Ũ } \\
\vdots \\
\dot{\alpha} \\
z \\
0 \\
z \\
\frac{a}{x} \\
\end{array}$} & 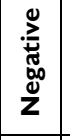 & 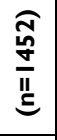 & $\underline{\underline{\underline{\Xi}}} \circ n-+n a$ & 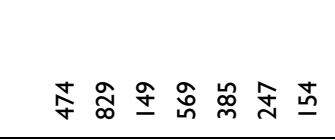 & 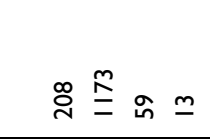 & 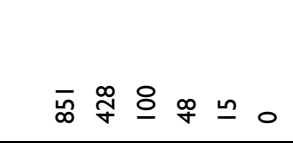 & ํํ \ั \\
\hline & 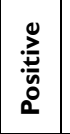 & 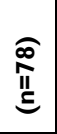 & $\simeq \begin{array}{c}n \\
N\end{array}$ & ๗ి & $+8 n-$ & $\pm \pm \sigma a \sim 0$ & $0 \leqslant$ \\
\hline \multicolumn{2}{|l|}{ a } & & 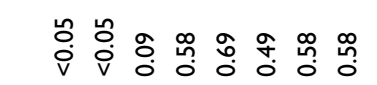 & 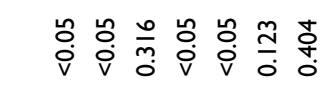 & 贻 웅 & 능 & :0 \\
\hline \multirow[b]{2}{*}{ 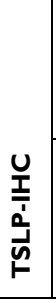 } & 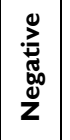 & 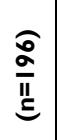 & $\underline{m} \stackrel{\infty}{=}+00-00$ & 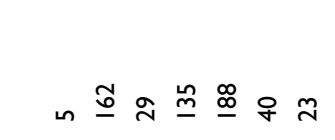 & $\infty \stackrel{\infty}{=} \infty \sim$ & $\overline{0} \underline{\underline{0}}=+0$ & $\stackrel{\circ}{\infty}$ \\
\hline & 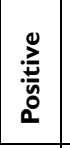 & 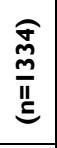 & $\underline{\underline{\Xi}} \stackrel{\cong}{=} \sim-m \sim r$ & హิ \$ & 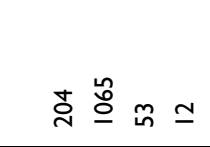 & 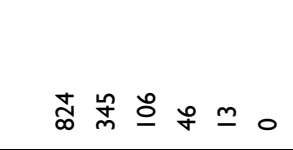 & $\stackrel{\mathscr{f}}{\underline{\infty}} \stackrel{\infty}{=}$ \\
\hline \multicolumn{2}{|l|}{ a } & & 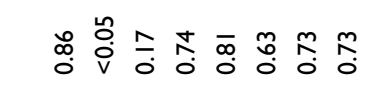 & 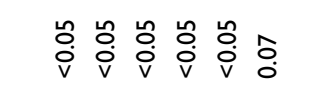 & 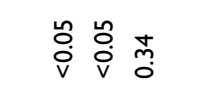 & 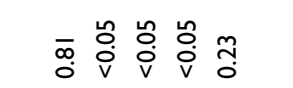 & กิ \\
\hline \multirow[b]{2}{*}{$\begin{array}{l}\frac{u}{\frac{1}{b}} \\
\frac{\hat{b}}{a}\end{array}$} & 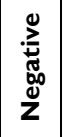 & $\frac{\frac{\sigma}{\sigma}}{\frac{\sigma}{J}}$ & 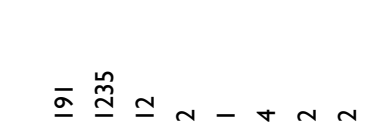 & 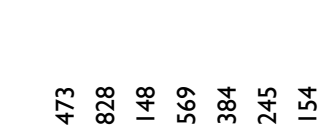 & 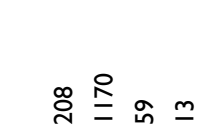 & 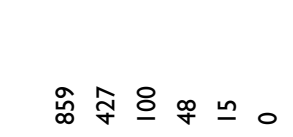 & 용 \\
\hline & \begin{tabular}{|l}
0 \\
\multirow{2}{*}{} \\
0 \\
0 \\
0
\end{tabular} & 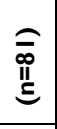 & $\underline{m}: \sim 00000$ & స 유 & $+m a-$ & 늉 & $0 \stackrel{n}{\kappa}$ \\
\hline \multicolumn{2}{|l|}{ 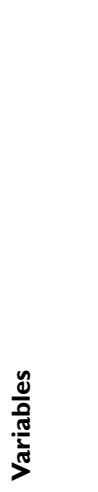 } & $\frac{\stackrel{0}{\hat{n}}}{\underline{\underline{n}}}$ & 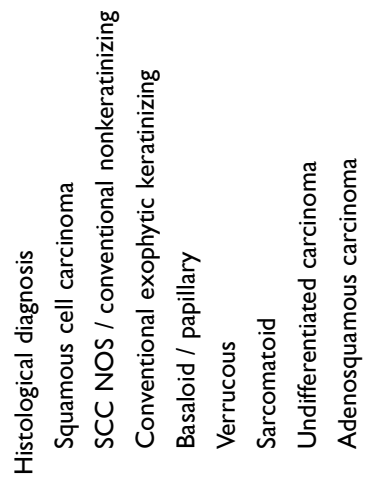 & 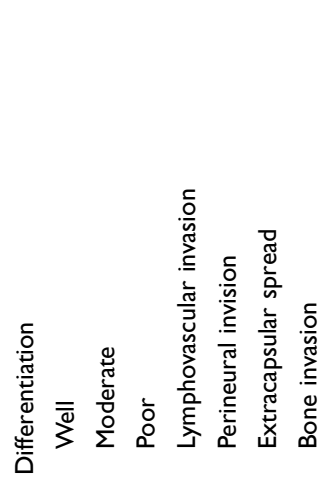 & 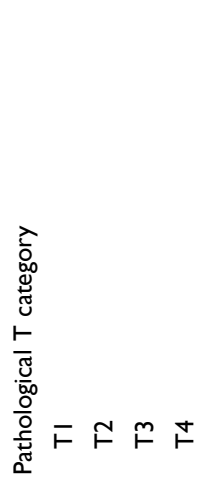 & 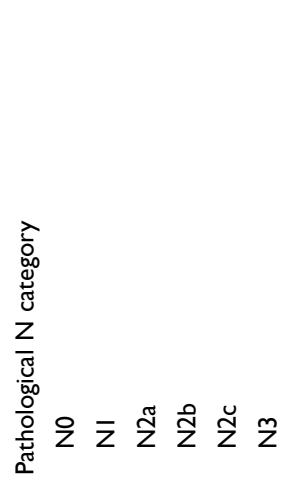 & 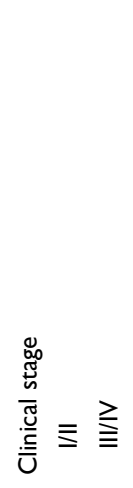 \\
\hline
\end{tabular}



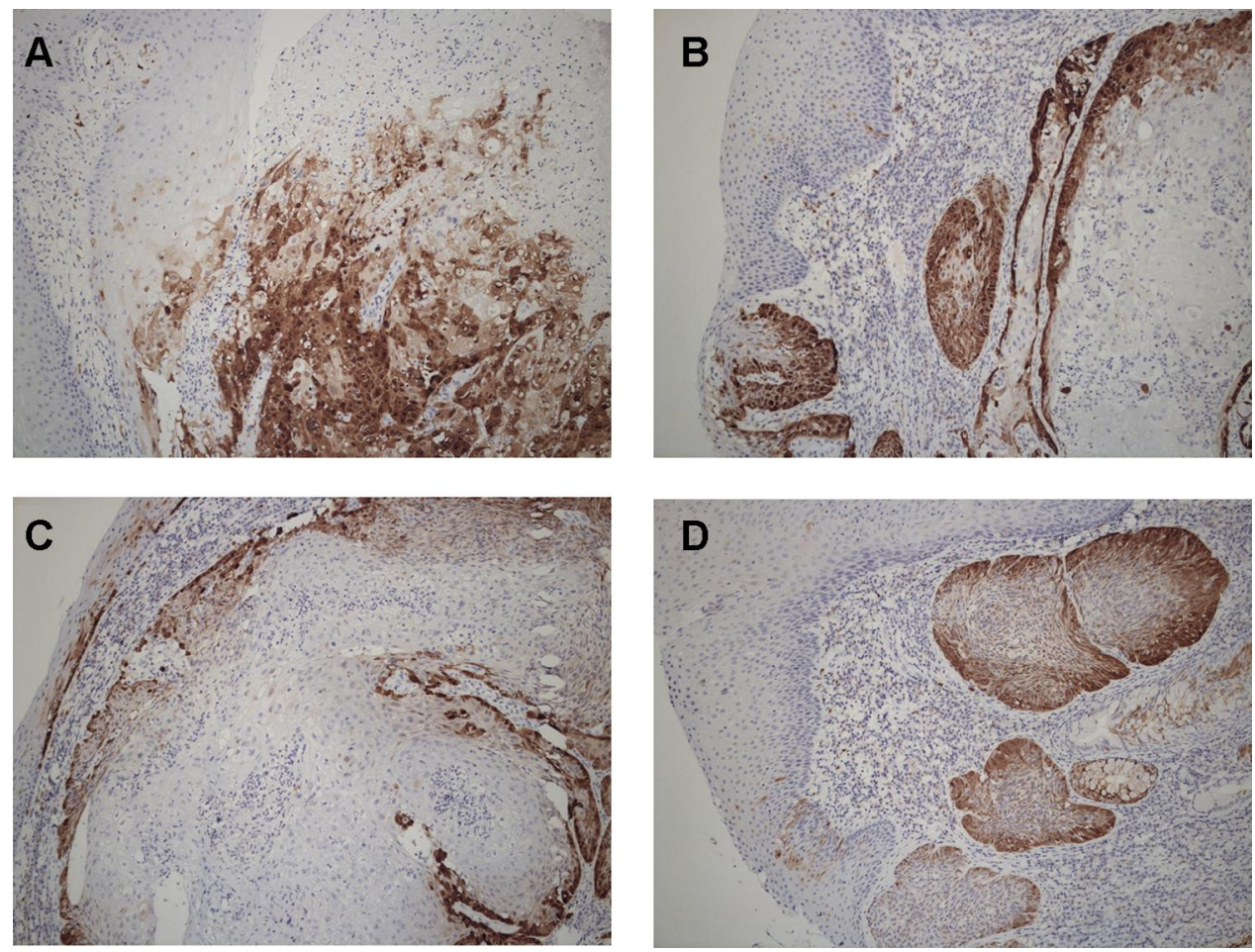

Figure I Expression of pl6 in oropharyngeal cancer cells. Immunohistochemistry observed pl6 expression in oropharyngeal cancer cells (magnification: 400). Cells with stained nuclei and cytoplasm are considered positive. Staining was scored as: (A) diffuse $(>80 \%$ of the cells were stained); (B) focal (small cell clusters, but $<80 \%$ of the cells were positive); (C) sporadic (isolated cells were positive, but < 5\%); (D) negative (< I\% of the cells were positive).
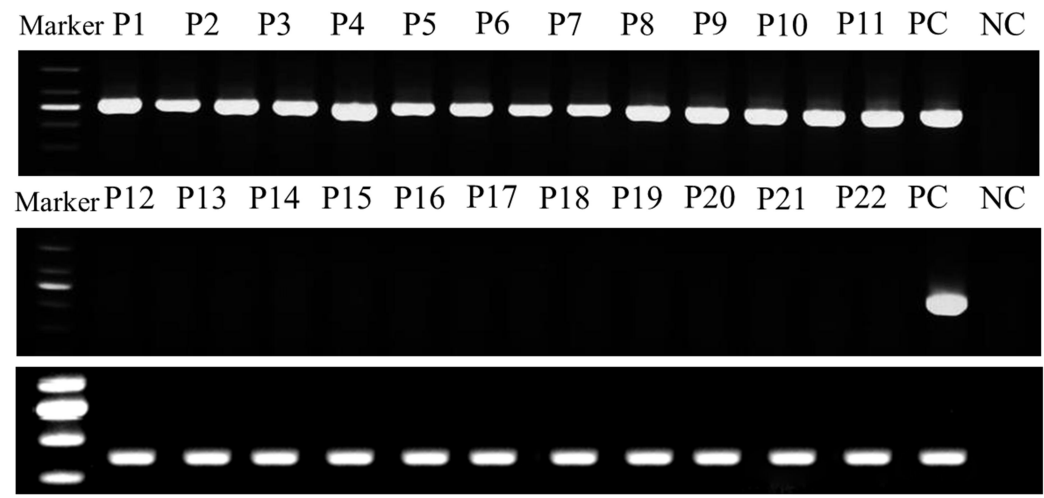

HPV

$\beta$-globin (250)

Figure 2 PCR analysis of HPV DNA in patients with oropharyngeal cancer. Using PCR, HPV status was determined in patients with oropharyngeal cancer.

\section{Prognostic Analysis of HPV and PI6 Status}

The positive expression of p16 in patient cells is associated with a significantly improved overall survival rate (OS, $\mathrm{P}=0.05)$. However, the results obtained through multivariate analysis are not clearly consistent. Our univariate analysis demonstrated that high clinical stage $(\mathrm{P}=$ 0.0475), high T stage ( $P=0.0002)$, age $(P=0.0058)$, HPV negativity $(\mathrm{P}=0.0029)$, p16 negativity $(\mathrm{P}=0.003)$, and TSLP positivity $(P=0.0001)$ were all significantly related to OS reduction. Multivariate analysis confirmed that only TSLP positivity $(\mathrm{P}=0.0004)$ was consistent with OS reduction and could be used as an independent risk factor.

\section{Discussion}

Previous studies suggested that patients with HPV-positive HNSCC had higher survival rates than HPV-negative patients. $^{2}$ In addition, scientific research indicated that HPV infection status can be used as a predictor of response to treatment, and is related to improved response to 

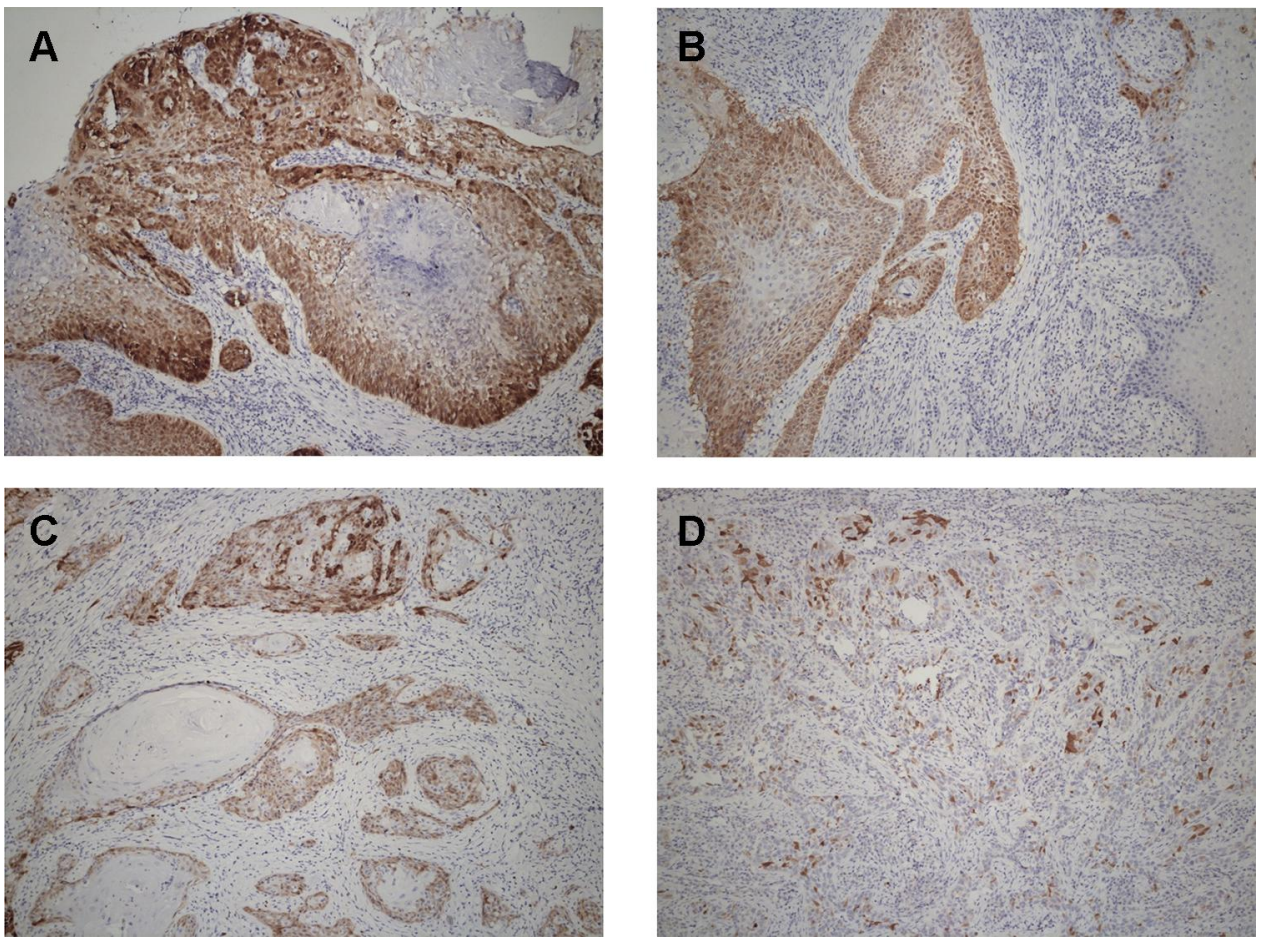

Figure 3 HPV-positive patients show more TSLP and TGF $\beta$ I-positive cells than HPV-negative patients. Immunohistochemical analysis of TSLP and TGF $\beta$ I in HPV-positive and HPV-negative patients. The number of TSLP and TGF $\beta$ I-positive cells was significantly greater in HPV-positive than in HPV-negative patients (magnification: $\times$ I00). (A) HPV(+) (PI6 positive) and TSLP presents a high expression state. (B) HPV(-) (PI6 negative) and TSLP presents a low expression state. (C) HPV(+) (PI6 positive) and TGF $\beta$ presents a high expression state. (D) HPV(-) (PI6 negative) and TGF $\beta$ I presents a low expression state.
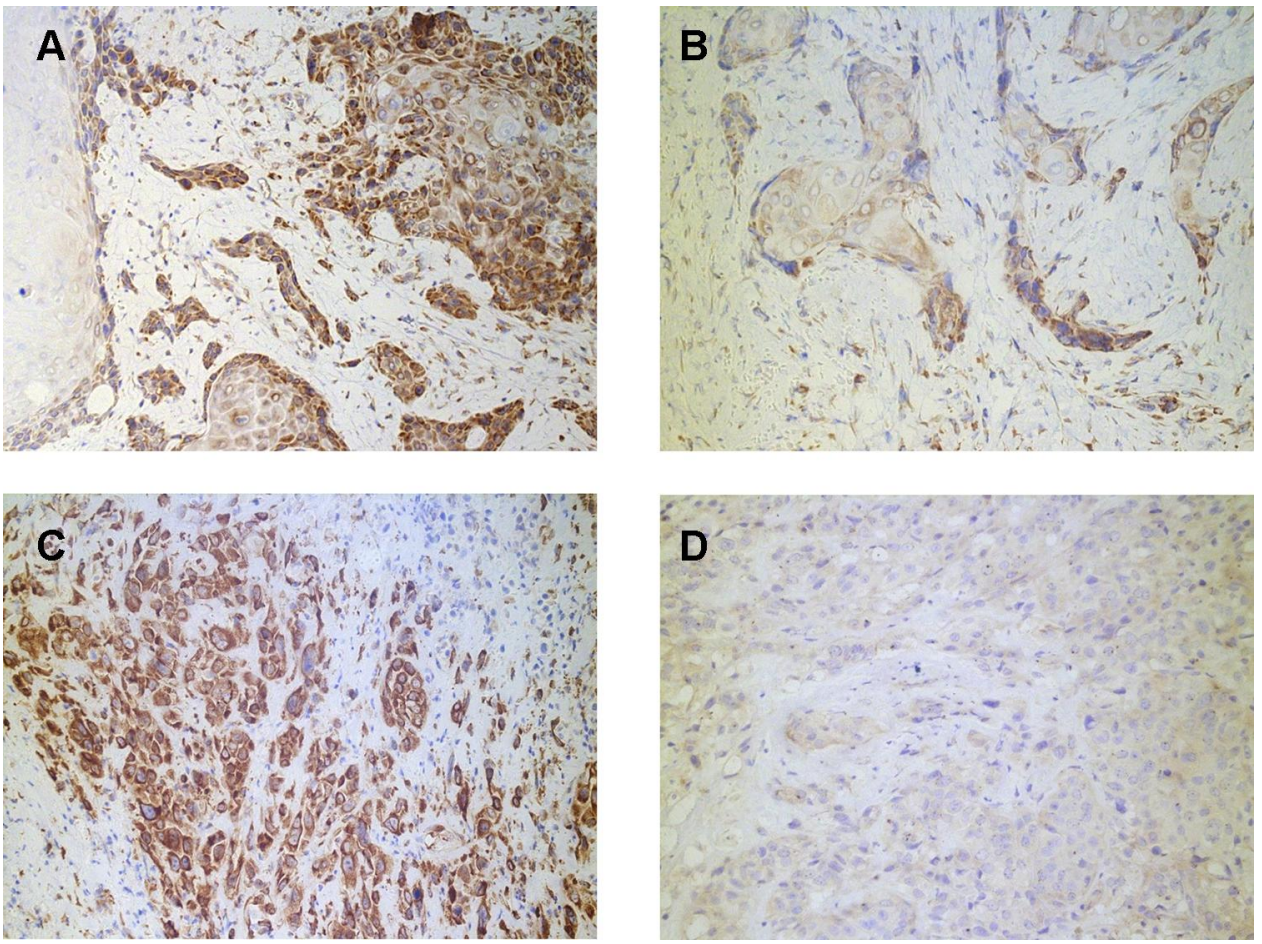

Figure 4 HPV-positive patients show more IFN $\gamma$ and OFDI-positive cells than HPV-negative patients. Immunohistochemical analysis of IFN $\gamma$ and OFDI in HPV-positive and HPV-negative patients. The number of IFN $\gamma$ and OFDI-positive cells was significantly greater in HPV-positive than in HPV-negative patients (magnification: $\times$ I00). (A) HPV $(+)$ (PI6 positive) and IFN $\gamma$ presents a high expression state. (B) HPV(-) (PI6 negative) and IFN $\gamma$ presents a low expression state. (C) HPV(+) (PI6 positive) and OFDI presents a high expression state. (D) HPV(-) (PI6 negative) and OFDI presents a low expression state. 


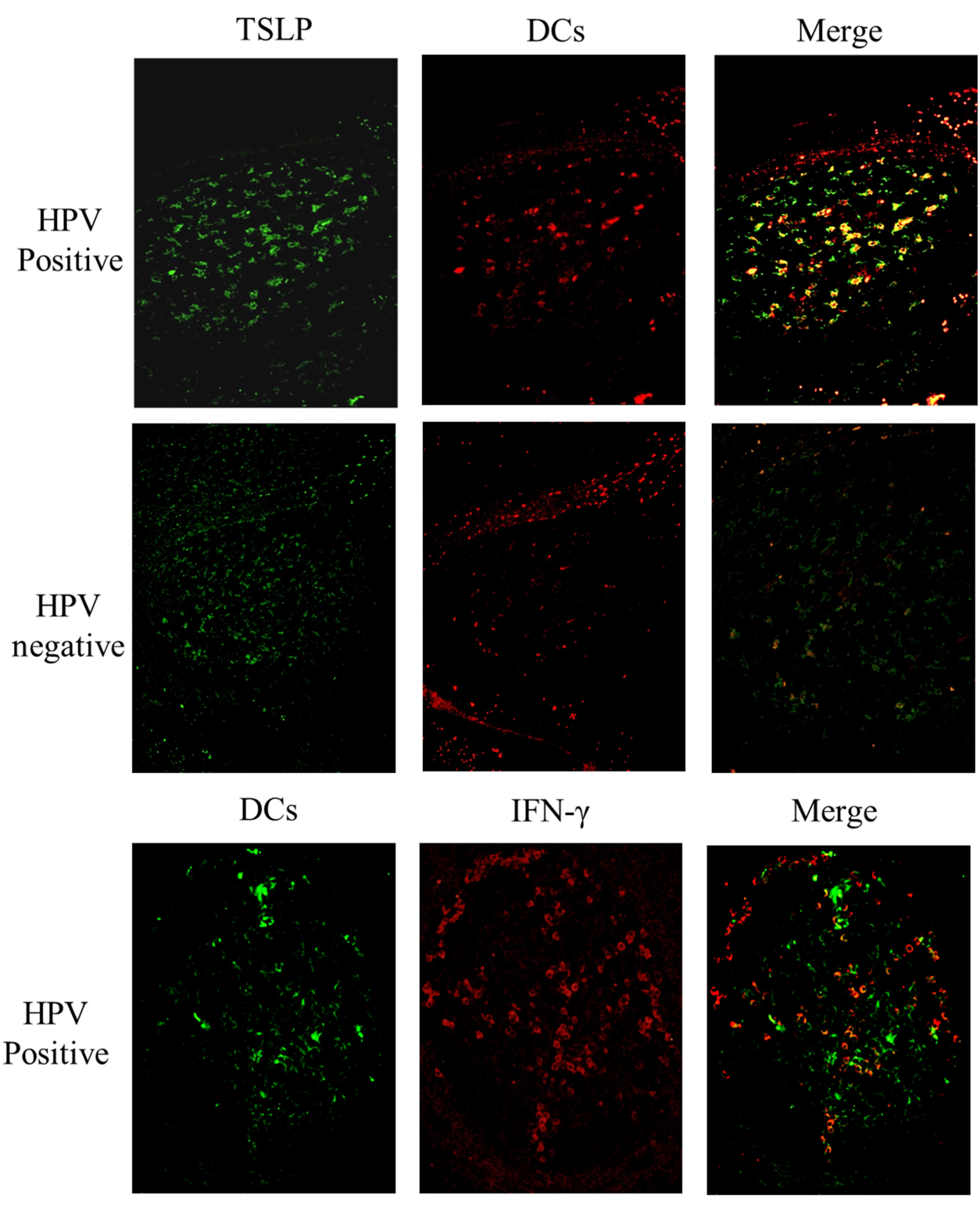

Figure 5 Coexpression of TSLP and IFN $\gamma$ with DC-SIGN is higher in HPV-positive patients. Double immunofluorescence for DC-SIGN (red) and TSLP (green), and DC-sign (green) and IFN $\gamma$ (red), showing double-positive cells (magnification: $\times 200$ ).

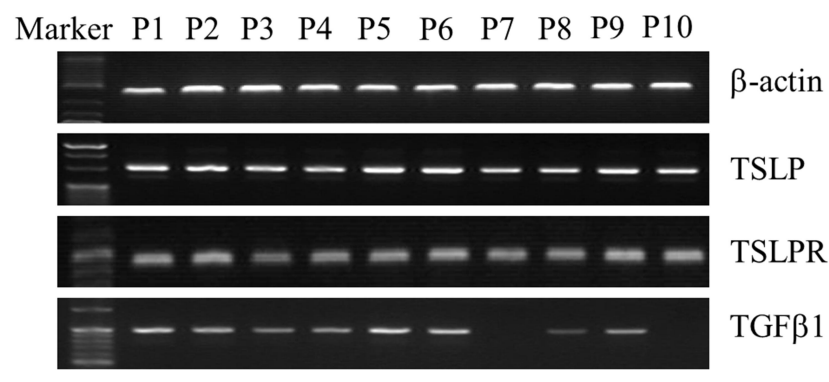

Figure 6 Increased expression of TSLP, TSLPR, and TGF $\beta$ I mRNA in HPV-positive patients. Using RT-PCR, mRNA levels of $\beta$-actin TSLP, TSLPR, and TGF $\beta$ I were detected in oropharyngeal tissue of HPV-positive and HPV-negative patients. Error bars indicate SEM. $P<0.05$ (Student's $t$-test) radiation therapy and chemotherapy. The study included 1530 patients in 16 years, and there are some inherent biases typical of retrospective cohorts. Although the use of adjuvant chemotherapy and high-conformal radiation technology has been increased clinically, the principle for patients to choose treatment methods is still mainly focused on surgical treatment and radiotherapy. Therefore, we use HPV-DNA testing as a gold standard and apply it clinically to evaluate the potential value of novel surrogate markers for HPV-induced cellular transformation. It is crucial to notice that our estimate of the 
HPV attribution score is much lower than the results of a recent meta-analysis of HPV in HNC: the oropharynx was $33.6 \%$, the $\mathrm{OC}$ was $22.2 \%$ and the larynx was $20.2 \%$ worldwide. $^{24}$ There are many reasons for this discrepancy, which may be due to the sample originating from different geographical locations, or it may be caused by the high heterogeneity of the procedures and steps used in the laboratory measurement and the measurement method.

HPV-AF estimates were also heterogeneous of in terms of sex and age at diagnosis. In our cohort, the estimated value of HPV-AF in men is significantly higher than that of women. This result is substantially different from the European population in other studies.Additionally, we also discover that the estimated value of HPV-AF is much higher in the age range of 46-55 years. Moreover, nearly $90 \%(86.67 \%)$ of the 1336 patients had a history of smoking, and nearly $90 \%$ (89\%) of patients with positive expression of p16 protein were used to smoking. In our cohort, there was a $60.13 \%(n=920)$ drinking rate, and nearly $70 \%$ of p16-positive patients drank alcohol.

It can be hypothesized that this huge heterogeneity of HPV-AFs reflects the obvious trend of geographical, temporal and demographic changes in people with smoking and oral HPV infection, leading to the rapid development of the epidemiology of HPV positive HNCs. We speculate that in our study cohort, tobacco smoking had a greater effect on oropharyngeal carcinogenesis than those that were almost universally caused by oral HPV infections in the 1960s and early 1980s, in which patients were approximately 46-55 years old. But the situation has changed since the 1980s. Although the number of tobacco smokers has decreased, the number of people who have been exposed to HPV in the oral cavity due to the prevalence of oral sex practices has increased. Therefore, the current prevalence and trend of HNC driven by HPV infection may virtually depend on these risk factors exposed 20-30 years earlier.

Previous studies have indicated that regardless of the HPV infection status in the oropharynx, p16 protein can independently serve as a predictor of radiotherapy response. ${ }^{6,7}$ Among patients undergoing surgery and postoperative adjuvant radiotherapy, the overall survival and disease-specific survival of patients with positive $\mathrm{p} 16$ protein were significantly longer than those with negative p16. ${ }^{8}$ The results shown in our univariate analysis are consistent with it. However, our multivariate analysis showed that $\mathrm{p} 16$ expression cannot be used as an independent predictor of survival. It can be reasonably assumed that p16 affects the survival of patients by affecting the invasive potential and proliferative capacity of the primary tumor. Nonetheless, scientists have fully affirmed the role of HPV and p16 as therapeutic targets and biomarkers in HNSCC.

In addition, we found that the protein and mRNA expression of TSLP in DCs was higher in HPV-positive than in negative patients, consistent with the fact that exogenous stimulation (including infection) can induce TSLP. Furthermore, the expression of OFD1 was markedly correlated with increased expression of TGF $\beta 1$ and IFN $\gamma$ in HPV-positive patients. Primary ciliogenesis was also reduced in HPV-positive patients. These results are consistent with former studies, that OFD1 is a key factor in the expression of the primary ciliogenesis in human cancer cells. We can speculate that HPV has caused changes in the immune system by affecting TSLP and dendritic cells, leading to changes in OFD1 and other indicators that affect the growth of ciliogenesis and affect the development of tumors.Additionally, TSLP protein expression correlates with that of TGF $\beta 1$, IFN $\gamma$, and OFD1 in HPV-positive OPSCCs, indicating that TSLP and primary ciliogenesis are closely related with the role of OFD1 in HPV-positive OPSCCs.

\section{Conclusion}

Our research indicates that the expression of $\mathrm{p} 16$ protein is related with early primary OPSCC and patients with P16 positive have longer survival after radiotherapy or surgery. In addition, the expression of p16 cannot be used as an independent predictor of survival. HPV infection can affect its invasive potential by promoting the expression of OFD1, whose expression affects the growth of primary ciliogenesis. TSLP induced by HPV infection is also possible to play a part in regulating primary ciliogenesis, which may modulate tumorigenesis and tumor invasion. Further research is required to clarify the role of HPV and TSLP in OPSCCs at the molecular level.

\section{Abbreviations}

FDC, follicular dendritic cell; FDC-SP, follicular dendritic cell secreted protein; IHC, immunohistochemistry; OPSCC, Oropharyngeal squamous cell carcinoma; HNSCC, head and neck squamous cell carcinoma; HPV, human papillomavirus; HPV-AF, HPV-attributable fraction; TSLP, thymic stromal lymphopoietin. 


\section{Ethical Approval}

All procedures performed in studies involving human participants were in accordance with the ethical standards of the institutional ethics committee of the Cancer Hospital Affiliated to Harbin Medical University and with the 1964 Helsinki declaration and its later amendments or comparable ethical standards.(2019-112)

\section{Informed Consent}

Informed consent was obtained from all individual participants included in the study.

\section{Acknowledgment}

This work was supported by the National Natural Science Foundation of China (82072985), the National Nature Science Foundation of Heilongjiang Province (YQ202 0H036), the Postdoctoral Scientific Research Developmental Fund of Heilongjiang Province (LBHQ18076), the N10 Found project of Harbin Medical University Cancer Hospital (2017-03), the Youth Elite Training Foundation of Harbin Medical University Cancer Hospital (JY2016-06), and the Outstanding Youth Foundation of Harbin Medical University Cancer Hospital (JCQN-2018-05), Special funds of central finance to support the development of local University (2019), WuJieping Medical Foundation (320.6750.19089-22, 320.6750.19089-48).

\section{Author Contributions}

All authors contributed to data analysis, drafting or revising the article, have agreed on the journal to which the article will be submitted, gave final approval of the version to be published, and agree to be accountable for all aspects of the work.

\section{Disclosure}

The authors declare that they have no competing interests.

\section{References}

1. Chow LQM. Head and neck cancer. $N$ Engl $J$ Med. 2020;382 (1):60-72. doi:10.1056/NEJMra1715715

2. Cramer JD, Burtness B, Le QT, Ferris RL. The changing therapeutic landscape of head and neck cancer. Nat Rev Clin Oncol. 2019;16:669-683.

3. Lechner M, Jones OS, Breeze CE, Gilson R. Gender-neutral HPV vaccination in the UK, rising male oropharyngeal cancer rates, and lack of HPV awareness. Lancet Infect Dis. 2019;19(2):131-132. doi:10.1016/S1473-3099(18)30802-8
4. Drolet M, Bénard E, Pérez N, Brisson M. HPV vaccination impact study group. Population-level impact and herd effects following the introduction of human papillomavirus vaccination programmes: updated systematic review and meta-analysis. Lancet. 2019;394 (10197):497-509. doi:10.1016/S0140-6736(19)30298-3

5. Worsham MJ, Chen KM, Datta I, et al. The biological significance of methylome differences in human papilloma virus associated head and neck cancer. Oncol Lett. 2016;12(6):4949-4956. doi:10.3892/ol.20 16.5303

6. Shaikh MH, Bortnik V, McMillan NA, Idris A. cGAS-STING responses are dampened in high-risk HPV type 16 positive head and neck squamous cell carcinoma cells. Microb Pathog. 2019;132:162-165. doi:10.1016/j.micpath.2019.05.004

7. Jalaly JB, Hosseini SM, Shafique K, Baloch ZW. Current status of p16 immunohistochemistry and HPV testing in fine needle aspiration specimens of the head and neck. Acta Cytol. 2020;64(1-2):30-39. doi:10.1159/000496158

8. Lechner M, Chakravarthy AR, Walter V, et al. Frequent HPV-independent p16/INK4A overexpression in head and neck cancer. Oral Oncol. 2018;83:32-37. doi:10.1016/j.oraloncology.2018.06.006

9. Stephen JK, Divine G, Chen KM, Chitale D, Havard S, Worsham MJ. Significance of p16 in site-specific HPV positive and HPV negative head and neck squamous cell carcinoma. Cancer Clin Oncol. 2013;2:51-61

10. Emmett S, Boros S, Whiteman DC, Porceddu SV, Panizza BJ, Antonsson A. Sexual behaviour, HPV status and p16 ${ }^{\mathrm{INK} 4 \mathrm{a}}$ expression in oropharyngeal and oral cavity squamous cell carcinomas: a casecase comparison study. J Gen Virol. 2018;99(6):783-789. doi:10. 1099/jgv.0.001069

11. Albers AE, Qian X, Kaufmann AM, Coordes A. Meta analysis: HPV and p16 pattern determines survival in patients with HNSCC and identifies potential new biologic subtype. Sci Rep. 2017;7(1):16715. doi:10.1038/s41598-017-16918-w

12. Gurín D, Slávik M, Shatokhina T, et al. Current perspective on HPV-associated oropharyngeal carcinomas and the role of p16 as a surrogate marker of high-risk HPV. Klin Onkol. 2019;32 (4):252-260. doi:10.14735/amko2019252

13. Li P, Zhang X, Gu L, Zhou J, Deng D, Chen Y-J. P16 methylation increases the sensitivity of cancer cells to the CDK4/6 inhibitor palbociclib. PLoS One. 2019;14(10):e0223084. doi:10.1371/journal. pone. 0223084

14. Munday JS, He YY, Aberdein D, Klobukowska HJ. Increased $\mathrm{p} 16^{\mathrm{CDKN} 2 \mathrm{~A}}$, but not $\mathrm{p} 53$, immunostaining is predictive of longer survival time in cats with oral squamous cell carcinomas. Vet $\mathrm{J}$. 2019;248:64-70. doi:10.1016/j.tvj1.2019.04.007

15. Ni Y, Zhang X, Wan Y, et al. Relationship between p16 expression and prognosis in different anatomic subsites of OSCC. Cancer Biomark. 2019;26(3):375-383. doi:10.3233/CBM-192402

16. Meng $\mathrm{H}$, Li $\mathrm{H}$, Ohe $\mathrm{R}$, et al. Thymic stromal lymphopoietin in tonsillar follicular dendritic cells correlates with elevated serum immunoglobulin A titer by promoting tonsillar immunoglobulin A class switching in immunoglobulin A nephropathy. Transl Res. 2016;176:1-17. doi:10.1016/j.trs1.2016.04.008

17. Borowski A, Vetter T, Kuepper M, et al. Expression analysis and specific blockade of the receptor for human thymic stromal lymphopoietin (TSLP) by novel antibodies to the human TSLPR $\alpha$ chain Cytokine. 2013;61(2):546-555. doi:10.1016/j.cyto.2012.10.025

18. Fabbri L, Bost F, Mazure NM. Primary cilium in cancer hallmarks. Int J Mol Sci. 2019;20(6):1336. doi:10.3390/ijms20061336

19. May-Simera HL, Wan Q, Jha BS, et al. Primary cilium-mediated retinal pigment epithelium maturation is disrupted in ciliopathy patient cells. Cell Rep. 2018;22(1):189-205. doi:10.1016/j.celrep.20 17.12 .038 
20. Tang Z, Lin MG, Stowe TR, et al. Autophagy promotes primary ciliogenesis by removing OFD1 from centriolar satellites. Nature. 2013;502(7470):254-257. doi:10.1038/nature12606

21. Huang SH, Xu W, Waldron J, et al. Refining American joint committee on cancer/union for international cancer control TNM stage and prognostic groups for human papillomavirus-related oropharyngeal carcinomas. J Clin Oncol. 2015;33(8):836-845. doi:10.1200/JCO.20 14.58.6412

22. Dahlstrom KR, Garden AS, William WN, Lim JMY, Sturgis EM. Proposed staging system for patients with HPV-related oropharyngeal cancer based on nasopharyngeal cancer $\mathrm{N}$ categories. J Clin Oncol. 2016;34(16):1848-1854. doi:10.1200/JCO.2015.64. 6448
23. Ang KK, Harris J, Wheeler R, et al. Human papillomavirus and survival of patients with oropharyngeal cancer. $N$ Engl $\mathrm{J}$ Med. 2010;363(1):24-35. doi:10.1056/NEJMoa0912217

24. Tumban E. A current update on human papillomavirus-associated head and neck cancers. Viruses. 2019;11(10):922. doi:10.3390/v11 100922

\section{Publish your work in this journal}

Pharmacogenomics and Personalized Medicine is an international, peer-reviewed, open access journal characterizing the influence of genotype on pharmacology leading to the development of personalized treatment programs and individualized drug selection for improved safety, efficacy and sustainability. This journal is indexed on the American Chemical Society's Chemical Abstracts Service (CAS). The manuscript management system is completely online and includes a very quick and fair peer-review system, which is all easy to use. Visit http://www.dovepress.com/testimonials.php to read real quotes from published authors. 